\title{
DTCWT-MBOFDM with Reconfigurable Down Converter for Smart Grid Communication
}

\author{
Duney Disney Sam ${ }^{1}$, Devappa Jayadevappa ${ }^{2}$ and Cyril Prasanna Raj Premkumar ${ }^{3}$ \\ ${ }^{1}$ Ph.D Scholar, VTU /, Sr. Assistant Professor, \\ New Horizon College of Engineering, Bangalore, India \\ ${ }^{2}$ Professor, Dep of E \& I, JSSATE, Bangalore, India \\ ${ }^{3}$ Dean $(R \& D), M S$ Engineering College, \\ Bangalore. India \\ 1duneydsam@gmail.com,2devappa.22@gmail.com \\ ${ }^{3}$ cyrilyahoo@gmail.com
}

\begin{abstract}
Smart meters in smart grid provide services such as meter information for billing, health monitor, phasor measurement, weather sensing and load characterization with outage detection and restoration. Communication between the load and the base station over smart grid is undertaken or monitored by smart meter. OFDM based communication module is an integral part of smart meter. In this paper, Dual Tree Complex Wavelet Transform (DTCWT) based Multi Band Orthogonal Frequency Division Multiplexing (MBOFDM) is designed to demonstrate the advantages of dual tree wavelet over that of wavelets for orthogonal multiplexing in terms of BER. Software reference model based on DTCWT, DWT, FFT and NCO are developed for comparison. The proposed DTCWTOFDM model achieves $17 \mathrm{~dB}$ improvement at 10-4 BER over that of other OFDM schemes. The time invariant property of dual tree wavelet also ensures improvement in BER performances. The reconfigurable down convert and up converter is reconfigurable and hence is designed for MBOFDM.
\end{abstract}

Keywords: DTCWT, OFDM, power line communication, digital down converter

\section{Introduction}

Smart gird technology is gaining importance in power generation, distribution and monitoring, over 1000 million smart grid users will be there world wide by 2022 [1]. In India there will be 130 million smart grid and smart meter users by 2021 . The major challenges in deploying smart grid technology are to upgrade existing infrastructure with smart meter for monitoring and control [2]. Use of power lines for communication monitored by smart meter requires use of high data rate communication technology such as OFDM to be designed for Power Line Communication (PLC). PLC is currently competing with technologies that are very much matured such as CAN, ProfiBus, DSL modems, LAN, Ethernet, Bluetooth, GSM and so on [3]. PLCs currently are operated in three bands of frequencies, low data rate $(9-148.6 \mathrm{KHz},<10 \mathrm{kbps})$, high data rate $(9-600$ $\mathrm{KHz}, 50 \mathrm{Kbps}-1 \mathrm{Mbps})$ and broadband $(1.5-60 \mathrm{MHz},>10 \mathrm{Mbps})$ [4]-[6]. The high data rate and broadband are based on OFDM standards and adopt stronger error correction schemes. Very recently Ultra Wide Band (50-578 MHz, $200 \mathrm{Mbps})$ are recommended for PLCs by IEEE 1901 standards with Home Plug versions. As the data rate over PLCs keep increasing from Kbps to hundreds of Mbps, the modulation schemes supporting high data rates also need to be redesigned to meet these challenges. One of the popular modulation

Received (April 18, 2017), Review Result (August 27, 2017), Accepted (September 8, 2017) 
schemes is the OFDM that forms the physical layer which decides the data rate of power line communication standards. Zhang et al [7] described wavelet-based multicarrier modulation with minimal PAPR and OFDM system as a special case of multicarrier modulation. Ma et al [8] analyzed the performance of OFDM system and found that multipath effect results in more adverse conditions than impulsive noise. Further the improvement of overall system performance by inclusion of the optimum guard interval was studied. Antony Jamin and Petri Mahonen [9] have discussed the use of Wavelet Packet Transform modulation as an alternative to OFDM in wireless communications. Additional features are those different wavelet families can be chosen for adjacent cells to avoid inter cell interference in mobile communication. Signal diversity is also possible with Wavelet Packet Transform. Depending upon the characteristics of the system such as link quality, impulse response and computational complexity, transform size is programmable with wavelet packets. MBOFDM is designed by replacing FFT-IFFT pair with DWT-IDWT pair, achieving improvement in BER results. In [10] it is reported that if the input signal has any abrupt time shifts the number of wavelet coefficients vary significantly leading to distribution of energy across sub bands. In [11] non-redundant wavelets that meet shift invariant property is discussed that modulate input signal leading to orthogonal modulation. In [12] Peak to Average Power Ratio (PARP) is computed for FFT based OFDM, Wavelet based OFDM and Complex Wavelet based OFDM, from the results presented it is found that complex wavelet based OFDM achieves best PARP performance with $3 \mathrm{~dB}$ improvement for 16 QAM with 64 sub carriers. In [13] [14] it is shown that polarization mode dispersion (PMD) is improved with use of dual tree wavelets, spectral efficiency and transmission distance is also improved with use of dual tree complex wavelets for OFDM. Dual tree complex wavelets consist of two filter banks that produce real and imaginary coefficients. In [15] DTCWT based OFDM model is developed using LeGall 5,3 filter, Antonini 9,7 filter, near symmetric 13,19 filter for the first filter bank and quarter sample orthogonal 10,10 filter is used for second stages of filtering. For PLCs the major challenges are down converting the modulated data to 50$578 \mathrm{MHz}$ signal for MBOFDM PLCs. In this paper design of DTCWT based OFDM discussed which is integrated with DDC for PLC applications. A novel architecture for MB-OFDM for PLC is designed and developed that can be used over power line achieving higher data rate. Section II discusses MB-OFDM architecture, section III discusses DTCWT architecture, section IV discusses software reference model design of MB-OFDM for power line communications and Section V is results and discussion and section VI is conclusion.

\section{Multiband OFDM System for PLC}

Important feature of UWB network is very low self interference, along with the wide bandwidth, thereby, large number of UWB terminals can be operated in a given area, either cooperatively as independent links or as part of ad hoc network. UWB is very tolerant to multipath because of the use of narrow pulses. When used in duplex link, accurate round trip delay and hence range is possible by the process of acquiring tight time synchronization. One approach to design an UWB system based on OFDM is to combine the multi-banding approach with a modulation technique as presented in Masood Ur Rehman et al [16] in which the spectrum is divided in to several sub-bands, whose bandwidth is approximately $500 \mathrm{MHz}$. The transmitted OFDM symbols are timeinterleaved across the sub-bands. An example of a multiband OFDM transmitter and receiver is presented in Batra et al [17] is shown in Figure 1 and the modulator is replaced with DTCWT. The upper side band is filtered and is taken through the UWB system to obtain the output data. Next section discusses the design and modeling of proposed MBOFDM UWB system with Digital Down Converter (DDC) and Digital Up Converter (DUC) for PLC. 


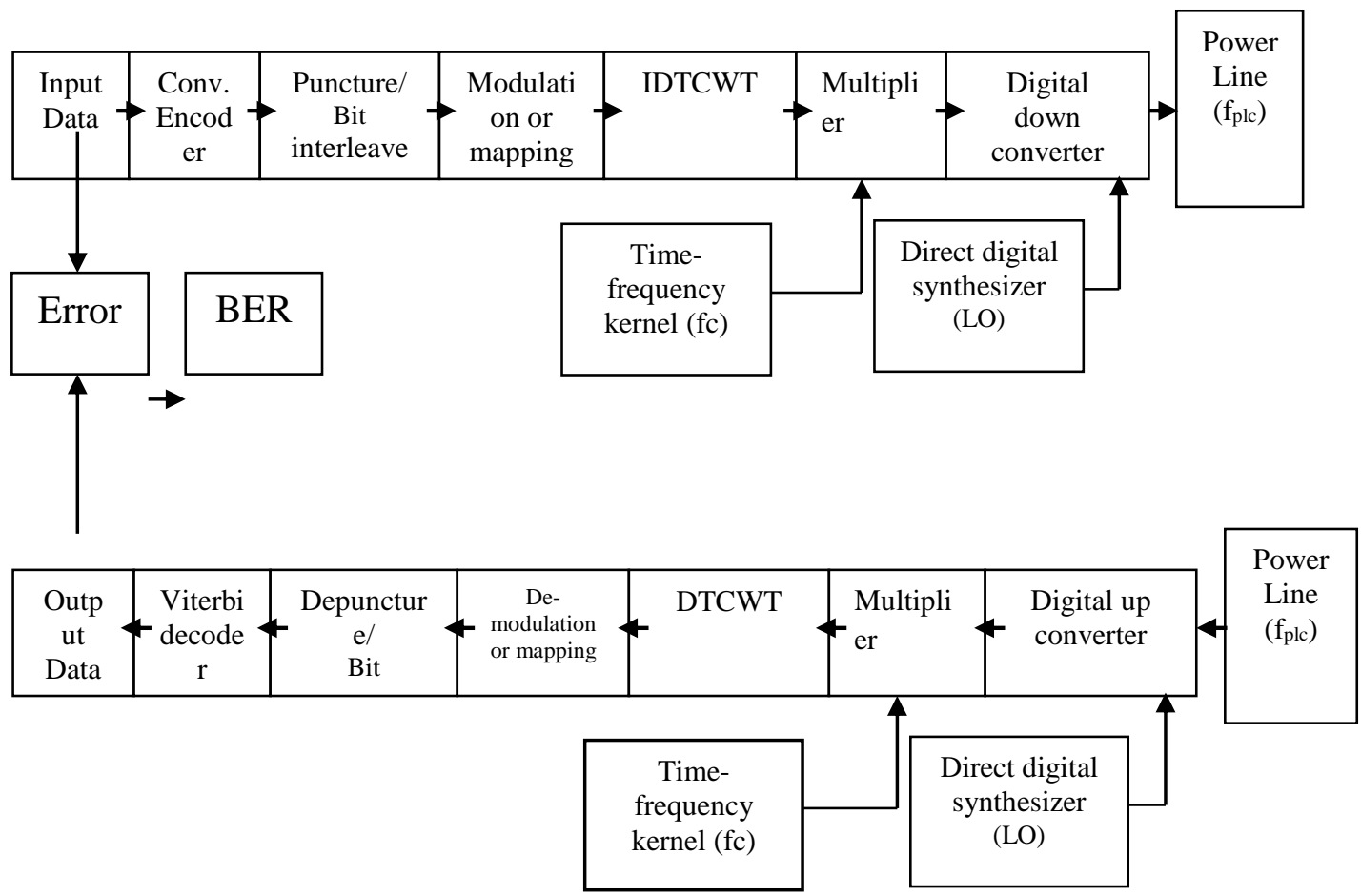

Figure 1. MBOFDM UWB with Digital Down Converter and Up Converter for PLCs

\section{Dual Tree Complex Wavelet Transform (DTCWT)}

DTCWT was introduced in [18], demonstrating the properties of shift invariance and directionality, suitability of complex wavelets for OFDM is also discussed in [19]. Signal analysis is carried out in DTCWT as similar to DWT, DTCWT consists of two separate decomposition trees as shown in Figure 2, among which the $\mathrm{H}$ filter (top filter bank) is the real and the $\mathrm{G}$ filter (bottom filter bank) is the imaginary tree. The $\mathrm{H}$ tree filter bank has low pass and high pass filters represented by h0 and h1 respectively, the $\mathrm{G}$ tree filter represented by g0 and g1 for low pass and high pass respectively. Figure 2 shows the DTCWT decomposition or forward DTCWT, with input signal y(n) decomposed to $5 \mathrm{sub}$ bands by the $\mathrm{H}$ tree and $\mathrm{G}$ tree with four stages. The first stage filter coefficients are not similar to succeeding stages of filter coefficients.

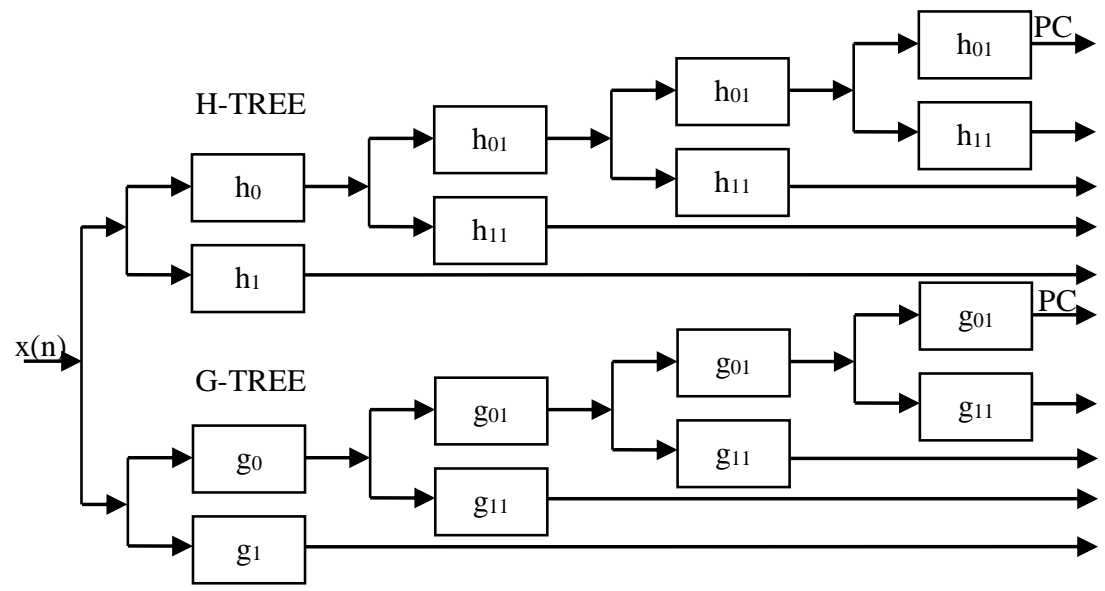

Figure 2. Kingsbury's Dual-Tree CWT 
The wavelet function relating the filter coefficients with wavelet function and scaling function are defined as in Equation (1) and Equation (2),

$$
\begin{aligned}
\Psi_{r}(t) & =\sqrt{2} \sum_{n} h 1(2) \phi_{r}(2 t-n) \\
\phi_{r}(t) & =\sqrt{2} \sum_{n} h 0(2) \phi_{r}(2 t-n)
\end{aligned}
$$

The filter coefficients $\mathrm{h} 1(\mathrm{n})$ and $\mathrm{h} 0(\mathrm{n})$ are related as in Equation (3), notation $\mathrm{r}$ is used for real transforms and equations (1) and (2) also represent imaginary functions with notation $r$ replaced with $i$.

$$
h 1(n)=(-1)^{n} h 0(d-n)
$$

Figure 3 shows the inverse DTCWT operation carried out with filter coefficients represented by h' and g'. The wavelet functions $\Psi \mathrm{r}$ and $\Psi \mathrm{i}$ are related by Hilbert transform as $\Psi \mathrm{r}=\mathrm{H}\{\Psi \mathrm{i}\}$, the complex wavelet function $\Psi(\mathrm{t})=\Psi \mathrm{r}+\Psi \mathrm{i}$. The low pass filters in the $\mathrm{H}$ tree and $\mathrm{G}$ tree need to satisfy half sample delay property [13] as given in Equation (4).

$$
G_{o}\left(e^{j \omega}\right)=e^{-j 0.5 \omega} H_{o}\left(e^{j \omega}\right)
$$

The expression in Equation (4) can be rewritten in terms of magnitude and phase function as shown in Equation (5).

$$
\begin{aligned}
& \left|G_{o}\left(e^{j \omega}\right)\right|=\left|H_{o}\left(e^{j \omega}\right)\right| \\
& \quad \angle\left(e^{j \omega}\right)=\angle H_{o}\left(e^{j \omega}\right)-0.5 \omega
\end{aligned}
$$

Similarly, the high pass filters in both trees need to satisfy half sample delay property. Selection of DTCWT filter coefficients need to satisfy half delay property, perfect reconstruction, good vanishing moments and linear phase. Design of DTCWT filters structure requires selection of appropriate filter coefficients for low pass and high pass filters, the filter structure processes the input to generate the real coefficients from which imaginary coefficients can also be obtained from the same input signal [15]. The first stage filter should be different from second stage filters [15].

\section{DTCWT Based OFDM}

The input data stream to be transmitted over communication channel is first modulated using QAM or QPSK modulation schemes to generate the real and imaginary symbols. The serial QAM symbols represented by $\left(\mathrm{a}_{\mathrm{i}, \mathrm{j}}\right)$ are grouped into frames of size $\mathrm{M}$, the serial to parallel converter converts each frames of $\mathrm{M}$ symbols into parallel data. The M QAM symbols are modulated to generate time-frequency subcarriers with the Inverse DTCWT (represented by $\Psi$ ) with $\mathrm{j}$ iterations and is represented as sum of discrete signals $\mathrm{x}[\mathrm{n}]$ as in Equation (6).

$$
x[n]=\sum_{i} \sum_{j=0}^{M-1} a_{i, j} \Psi_{j}[n-i M]
$$

OFDM is carried out with the structure shown in Figure 3, the structure consists of four stages of filter banks represented by filter coefficients $\hat{h}_{0}, \mathrm{~h}_{1}{ }_{1}$ and $\mathrm{g}_{0}, \mathrm{~g}_{1}{ }_{1}$. QAM signal consisting of real and imaginary data is modulated simultaneously with the $\mathrm{H}$ tree filter bank and $G$ tree filter bank to generate the OFDM signal $x[n]$. At the receiver, demodulation is carried out with the filter bank structure shown in Figure 2. The received signal $\mathrm{y}[\mathrm{n}]$ is processed by four stages of filter bank architecture to demodulate the real and imaginary QAM symbols from the modulated sub carriers. The QAM symbols that are in serial are passed through a demultiplexer unit that has $\mathrm{M}=2^{\mathrm{N}}$ output lines, with $\mathrm{N}$ 
select lines. An N-bit counter is used to generate $2^{\mathrm{N}}$ counts to demultiplex the serial data into $\mathrm{M}$ symbols and is stored in a register of size $\mathrm{M}$. Both the real and imaginary QAM symbols are processed independently to generate parallel $\mathrm{M}$ symbols and are stored in an output register. The output register is connected to the $\mathrm{H}$ tree and $\mathrm{G}$ tree of inverse DTCWT as shown in Figure 3. The binary bit stream (bi) is de-multiplexed and is modulated, the modulated data comprising of real and imaginary data output is multiplexed and transmitted.

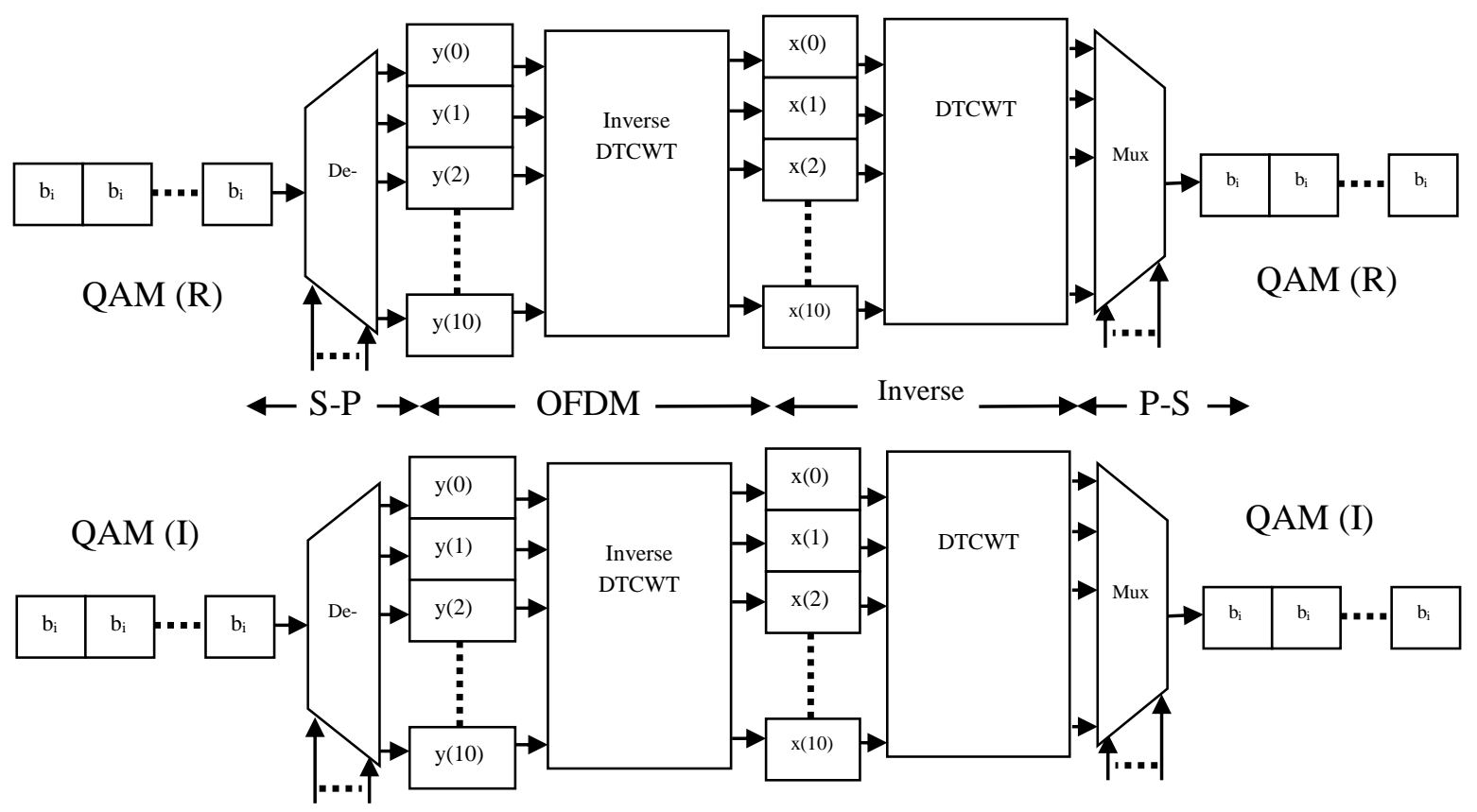

Figure 3. OFDM Modulator \& De-Modulator for Real and Imaginary Channel

The QAM symbols represented by bi, $\mathrm{i}=1,2 \ldots \mathrm{M}$ are modulated by $\Psi(\mathrm{t})($ Scaling function) and is represented as in Eq. (7) and (8) representing real and imaginary signal after orthogonal modulation,

$$
\begin{aligned}
& \operatorname{Re}[x(n)]=b_{1 k} \Psi_{1, k}(n)+\sum_{j=2}^{\frac{M}{2}} b_{j, k} \Psi_{j, k}(n) \\
& \operatorname{Im}[x(n)]=b_{\frac{N}{2}+1} \Psi_{1, k}(n)+\sum_{j=\frac{N}{2}+1}^{\frac{M}{2}} b_{j, k} \Psi_{j, k}(n)
\end{aligned}
$$

At the receiver, the received signal is de-multiplexed into real and imaginary components and is demodulated by forward DTCWT to obtain real and imaginary QAM symbols. Perfect reconstruction of modulated data is possible with synchronisation between transmitter and receiver.

A matrix operation $x=W b_{i}$, where input symbols $b_{i}$ are transformed to output data represented by $x$, can be used to represent the mathematical operation of DTCWT. As DTCWT is complex the $\mathrm{W}$ matrix consists of real and complex wavelets represented by $\mathrm{W}_{\mathrm{R}}$ corresponds to wavelet matrix for real DTCWT and $\mathrm{W}_{\mathrm{I}}$ corresponds to wavelet matrix for imaginary DTCWT, the DTCWT coefficient matrix can be written as in Equation (9) [20],

$$
W=\frac{1}{\sqrt{2}}\left(\begin{array}{cc}
I & j I \\
I & -j I
\end{array}\right)\left[\begin{array}{l}
W_{R} \\
W_{I}
\end{array}\right]
$$


The inverse DTCWT coefficient matrix can be written as in Equation (10) [20],

$$
W^{\prime}=W^{-1}=\frac{1}{\sqrt{2}}\left[W_{R}^{-1} W_{I}^{-1}\right]\left(\begin{array}{cc}
I & I \\
-j I & j I
\end{array}\right)
$$

In this work, bior6.8 wavelet is chosen for DTCWT computation, the filter coefficients for first stage dual tree (bior6. ','), which consists of 18 low pass filter coefficients and 11 high pass filter coefficients for $\mathrm{H}$ tree and $\mathrm{G}$ tree. The DTCWT decomposes the input signal into four outputs in the first stage in terms of mother wavelet $\Psi$ and scaling function $\Phi$. For computation of inverse DTCWT the transpose of the forward filter coefficients are used.

\section{Design of DDC and DUC for MBOFDM UWB}

The block diagram of the DUC and DDC is as shown in Figure 4 and Figure 5 respectively. The baseband signal is translated to desired channel using the DDS and mixer comprising the multipliers. For multiband OFDM, the DDC and DUC are designed to be reconfigurable operate at frequencies of $24 \mathrm{KHz}, 48 \mathrm{KHz}, 96 \mathrm{KHz}$, $196 \mathrm{KHz}$ and $400 \mathrm{KHz}$. The NCO designed to generate the designed carrier frequency for reconfiguration.

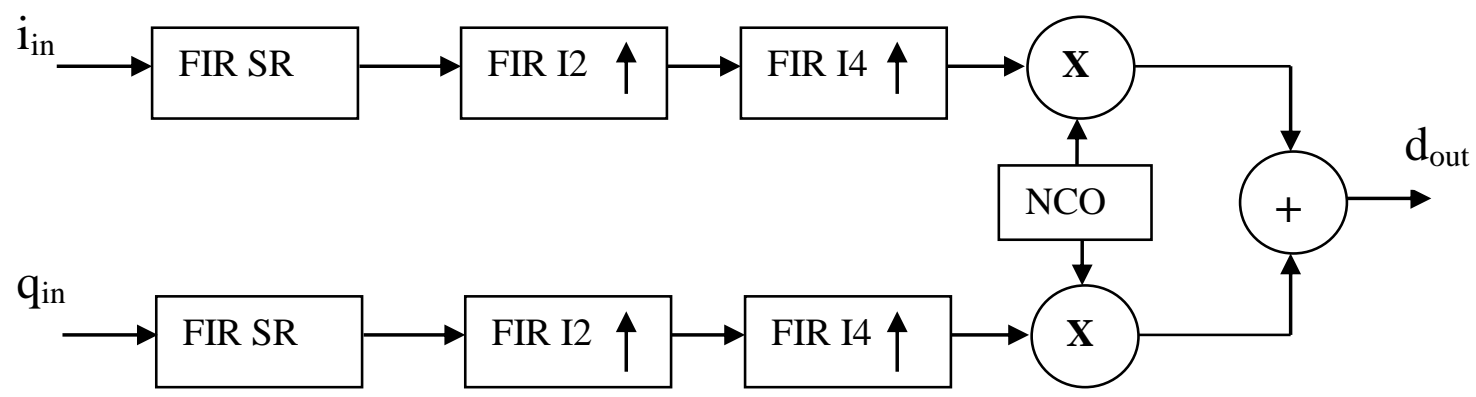

Figure 4. Reconfigurable DUC

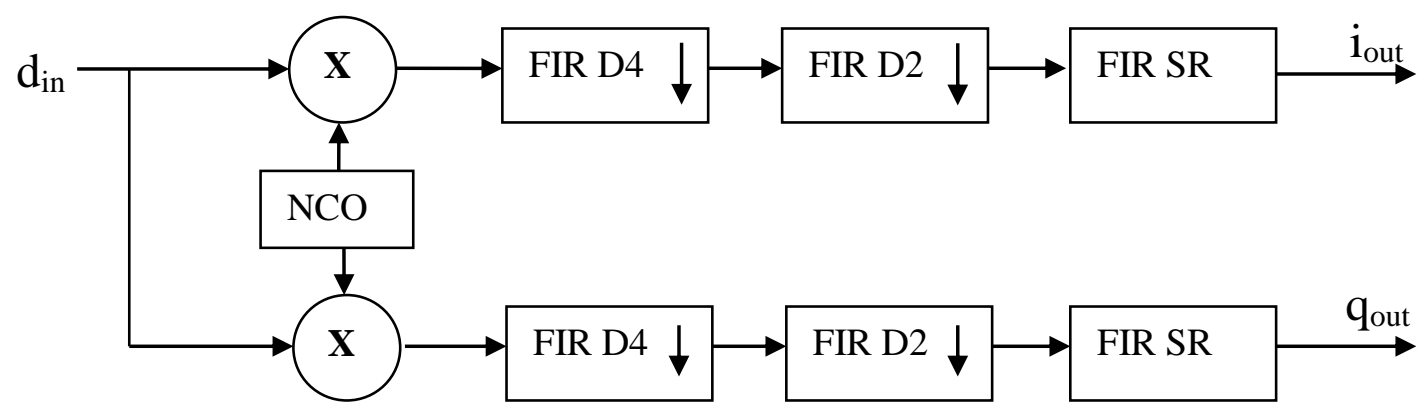

Figure 5. Reconfigurable DDC

The designed reconfigurable DUC and DDC are designed and modeled in Verilog HDL and simulated in ModelSim. The DUC and DDC pair is simulated with $4 \mathrm{KHz}$ input signal for validation of its functionality and the results are presented in Figure 6. The OFDM modulator designed with DTCWT which is developed in Simulink environment is integrated with DUC-DDC pair model developed with Hardware-Software co-simulation model in Matlab environment to evaluate the SNR and BER performances. 


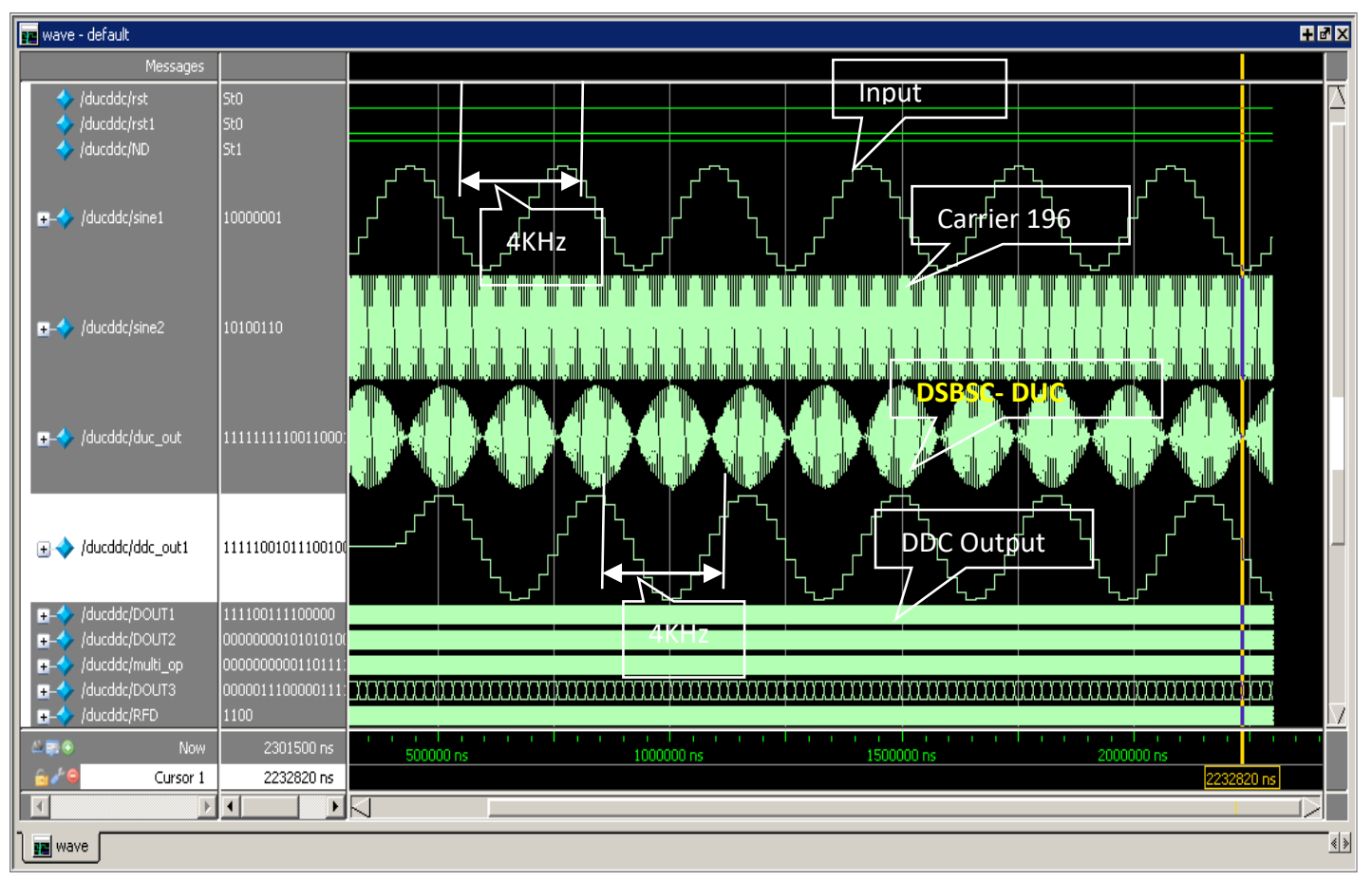

Figure 6. DUC and DDC for Carrier $196 \mathrm{KHz}$

\section{Results and Discussion}

The developed Simulink model is simulated with MATLABR13 assuming the channel to be of multipath Rayleigh distributed model with AWGN. The BER results of OFDMDTCWT are compared with that of OFDM-NCO, OFDM-FFT and OFDM-DWT. The software model developed considers an input data which is a random number consisting of integer sequences that forms the input to OFDM model. Forward Error Correction (FEC) code consisting of convolutional codes with rate $1 / 2$ is chosen. Data interleaving is carried out to improve data rate. The modulation technique that can be used is BPSK, QPSK, 16-QAM and 64-QAM. The real and imaginary symbols generated by modulation scheme are converted to parallel data symbols. 256 point FFT is used, that modulates the parallel symbols both in real and imaginary pair with inverse FFT. The OFDM scheme is designed to support 192 sub carriers, with 8 pilot carriers. 56 dummy symbols are introduced that performs cyclic prefix to make the QAM symbols of size 256 , which is modulated using 256 point inverse FFT. The modulated data is transmitted over AWGN channel with options to choose channel parameters such as fading, non-fading, flat-fading and or dispersive multipath fading. The receiver consists of channel estimation model, hard decision demodulation, deinterleaving, Viterbi decoding and Reed-Solomon decoding. BER is computed and compared for various OFDM techniques. The FFT based OFDM model, NCO-based OFDM model, DWT based OFDM model and DTCWT based OFDM model are compared with regard to BER with common modulation schemes such as 8-QAM and AWGN channel. For wavelet based model bior4.4 is used as wavelet function. The BER against bit energy to noise power spectral density ratio $(\mathrm{Eb} / \mathrm{No})$ of the developed systems were analyzed in AWGN, flat fading, and frequency-selective fading channels, the channel is assumed to have uniform error across the channel. Figure 7 presents the performance of the proposed OFDM-DTCWT model compared with all other models. BER vs Eb/No plot for varying in Eb/No is also compared with ideal OFDM. 


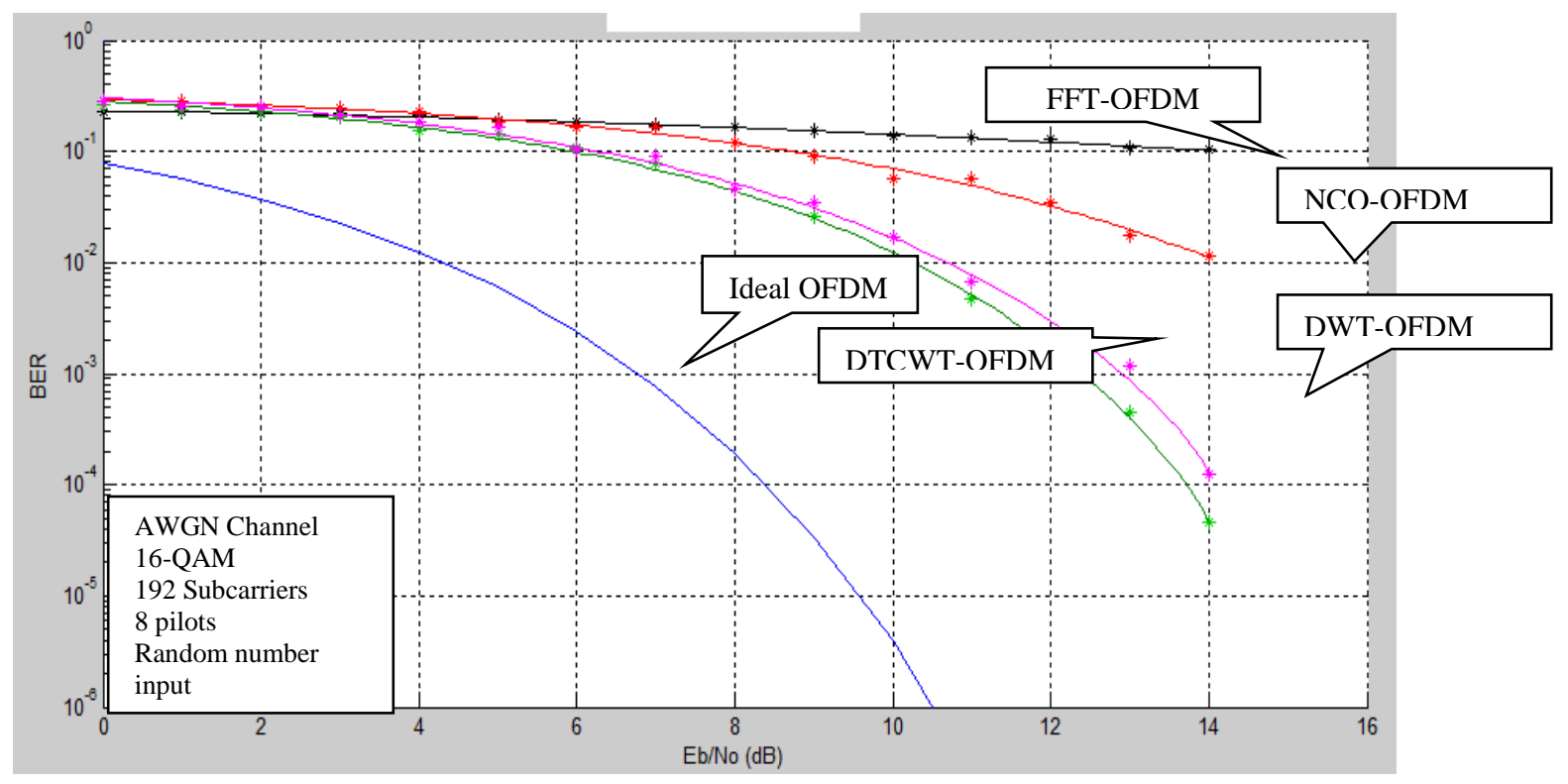

Figure 7. BER Comparison of OFDM Schemes

From the BER performances, it is found that DTCWT based OFDM performs better in terms of other schemes. BER $=10-4$ resulted in $17 \mathrm{~dB}$ improvement for the proposed system compared with FFT based OFDM system. BER $==10-4$ resulted in $4 \mathrm{~dB}$ improvement over DWT based OFDM system.

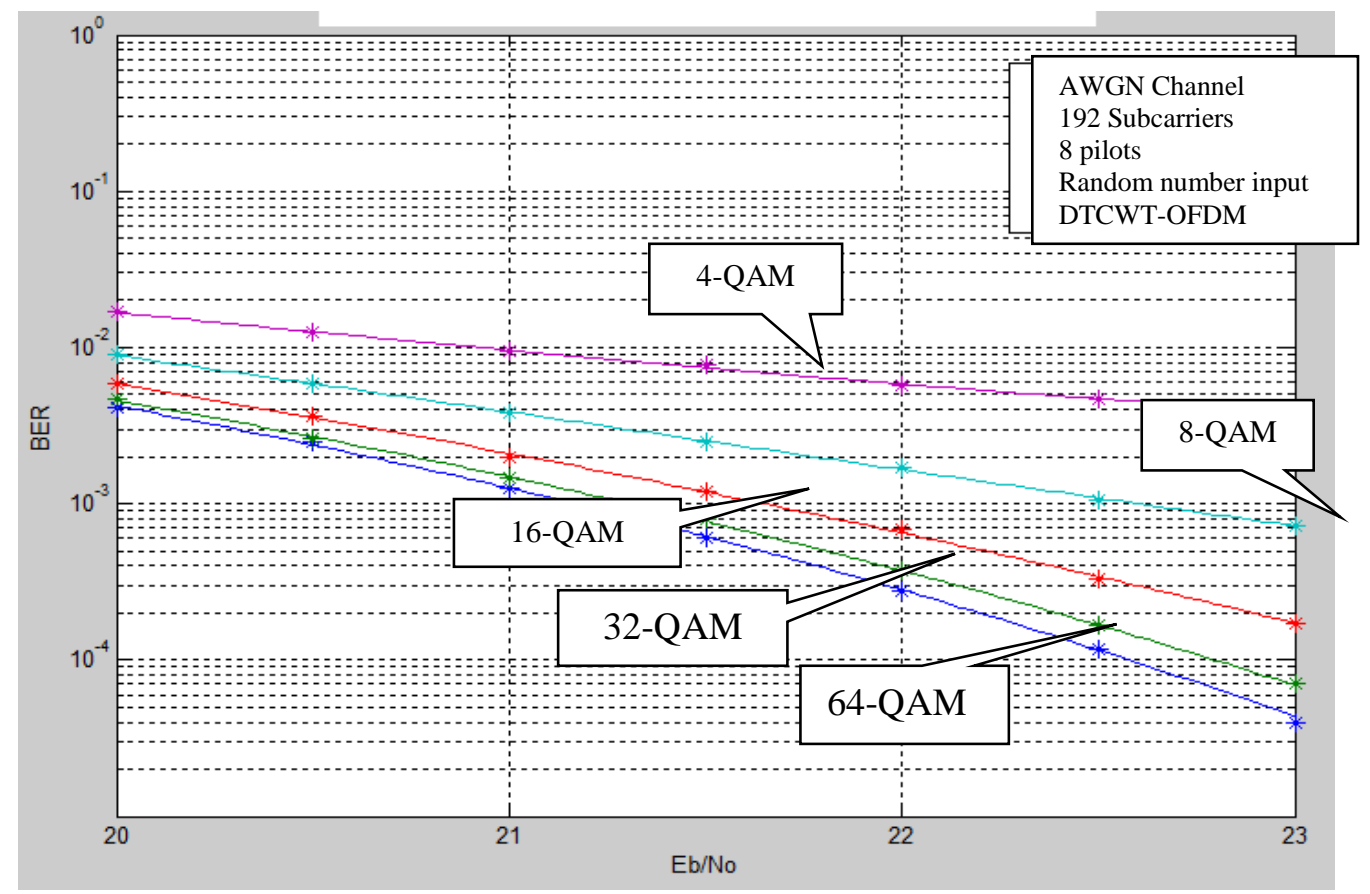

Figure 8. DTCWT-OFDM with Different Modulation Schemes

Figure 8 show the performance of the proposed system in comparison of DTCWT based OFDM with 4-QAM, 8-QAM, 16-QAM, 32-QAM and 64-QAM in a flat Rayleigh fading channel as well as AWGN. At 10-4 BER there is an improvement in $14 \mathrm{~dB}$ for $64-$ QAM based DTCWT-OFDM over that of 4-QAM based DTCWT-OFDM. Figure 9 shows the frequency spectrum of DTCWT-OFDM signal with 192 sub carrier frequencies. 


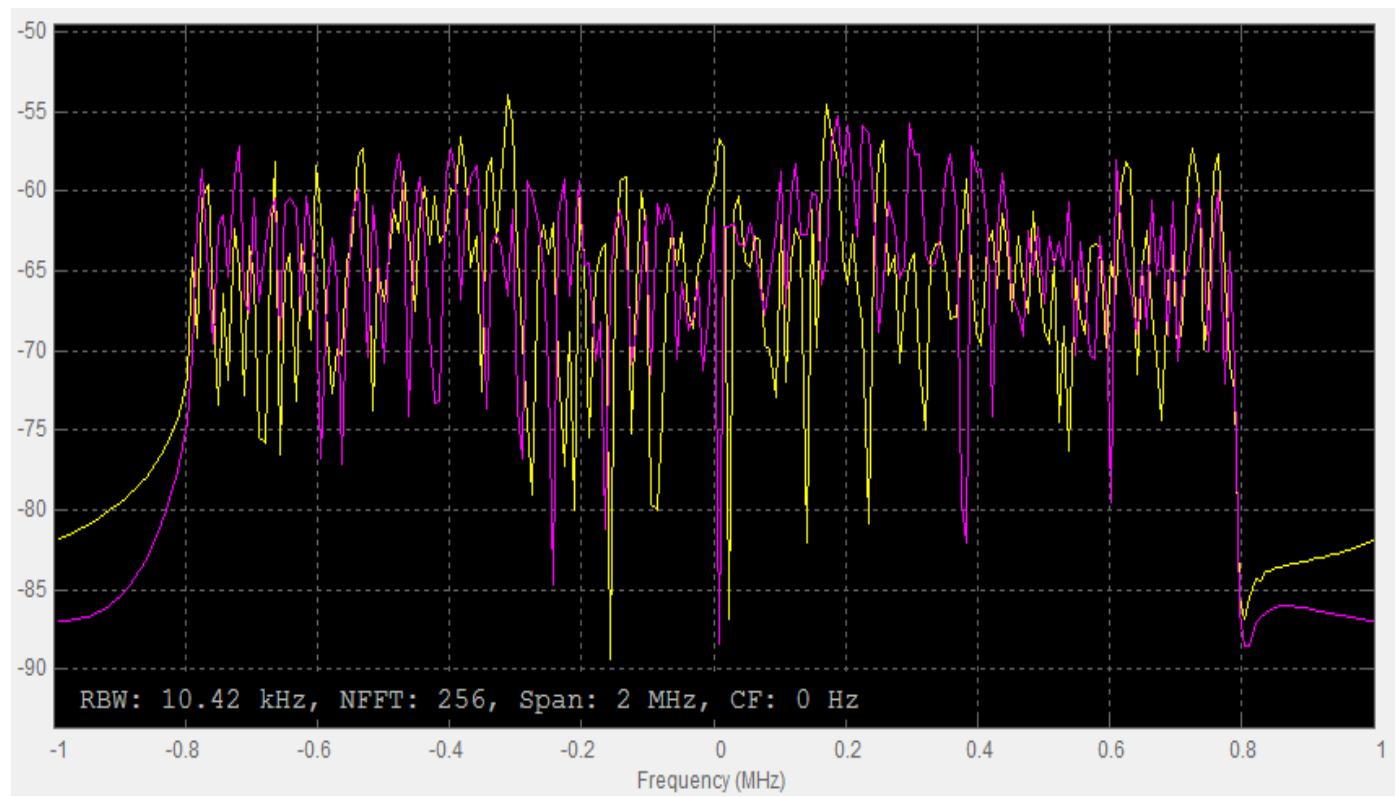

Figure 9. Frequency Spectrum of DTCWT-OFDM

The DTCWT-OFDM is down converted to frequency band $50 \mathrm{MHz}-528 \mathrm{MHz}$ with the use of digital down converter as discussed in previous section. Figure 10 shows the frequency spectrum of down converted OFDM signal for PLC.

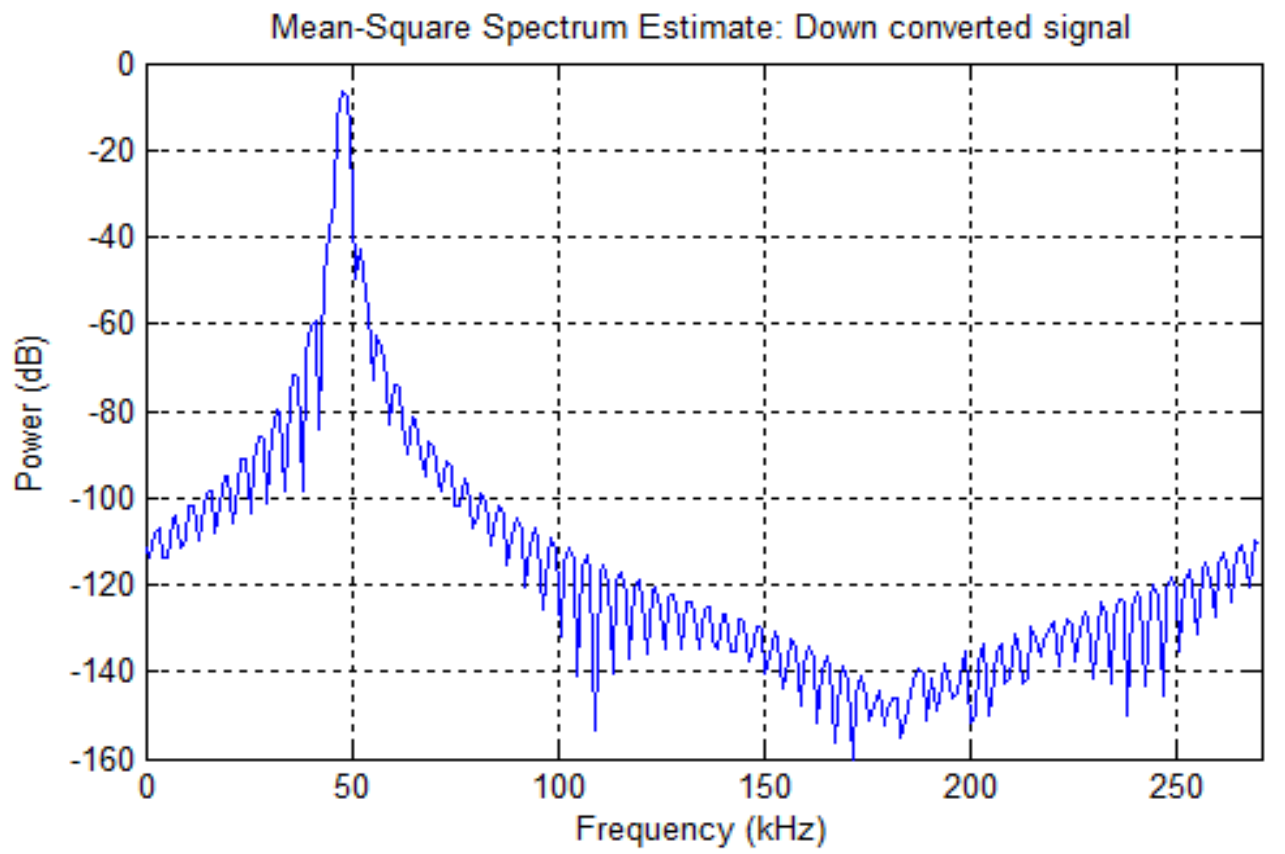

Figure 10. Down Converted OFDM Spectrum

The decimal values at the output of DDC in Simulink are taken. SNR is calculated and is found to be $125 \mathrm{~dB}$. THD is calculated by considering the first five harmonics and is found to be 1.281. From the THD value it is found that the harmonic component is not very dominant in the UWB system over PLC. The major challenges are the down conversion of $3939 \mathrm{MHz}$ centre frequency to $52 \mathrm{MHz}$ component as per PLC requirement. The down conversion leads to harmonics and sub components that need to be filtered using suitable filters that introduce minimum harmonics. On the other hand at 
the receiver side, the digital up converter need to up convert the signal from $52 \mathrm{MHz}$ to $3939 \mathrm{MHz}$, which again introduces harmonics and sub components. Optimum design of CIC filters and PFIR filters aids to overcome the challenges in down conversion and up conversion. The frequency band for PLC between $50 \mathrm{MHz}$ to $578 \mathrm{MHz}$ need to be reconfigurable, hence there is need for reconfigurable DDC which need to be designed as LO module.

\section{Conclusion}

In this paper, DTCWT-MBOFDM system is designed and is integrated with digital down converter at the transmitter and digital up converter at the receiver to transmit data over power line in smart grid environment. The designed system supports data rate in the range of $50 \mathrm{Mbps}$ to $500 \mathrm{Mbps}$, the input data is translated to UWB frequency in the range between $3939 \mathrm{MHz}$ to $8190 \mathrm{MHz}$ and is further down converted to the range $50 \mathrm{MHz}$ to $578 \mathrm{MHz}$ for power line requirement. The system is modeled using, the integrated system is simulated for various test cases of input data by modeling the system in Matlab and Simulink. Harmonic distortion is found to be of 1.2, as CIC and PFIR filters are used to minimize harmonic distortion in digital up converter and down converter. The design is suitable for power line communication. Hardware complexities and feasibility of the proposed system for smart meter applications on FPGA platform is recommended for real time applications.

\section{References}

[1] B. Hoang, "Smart Grids", published on the IEEE Emerging Technology portal, 2006-2012. http://www.ieee.org/go/emergingtech

[2] U. Pérez, N. Hernández, L. de 1, D. Vega and I. Angulo, "State of the Art and Trends Review of Smart Metering in Electricity Grids", Appied Science, vol. 6, no. 3, (2016), pp. 68.

[3] ITU Recommendation G.9960, Unified high-speed wireline based home networking transceivers Foundation, (2009).

[4] IEEE P1901 Draft Standard, Broadband over Power Line Networks: Medium Access Control and Physical Layer Specifications, (2010).

[5] N. Pavlidou, A. H. Vinck, J. Yazdani and B. Honary, "Power line communications: State of the art and future trends", IEEE Communication Mag., vol. 41, no. 4, (2003), pp. 34-40.

[6] A. Majumder and J. Caffery, "Power line communications: An overview", IEEE Potentials, vol. 23, no. 4, (2004), pp. 4-8.

[7] "HomePlug Powerline Alliance", HomePlug AV White Paper. [Online]. Available: http://www.homeplug.org.

[8] H. Zhang, Y. Dongfeng and Matthias, "Novel Study on PAPRs Reduction in Wavelet-Based Multicarrier Modulation Systems", Computer Journal of Elsevier Digital Signal Processing, vol. 17, no. 3, (2007), pp. 272-279.

[9] Y.H. Ma, P.L. So and E. Gunawan, "Performance analysis of OFDM systems for broadband power line communications under impulsive noise and multipath effects", IEEE Trans-actions on Power Delivery, vol. 20, no. 2, (2005), pp. 674-682.

[10] A. Jamin and P. Mahonen, "Wavelet Packet Modulation for Wireless Communications", Wireless Communications \& Mobile Computing Journal, vol.5, no. 2, (2005), pp.1-18.

[11] M. Guatier, J. Lienard and M. Arndt, "Efficient Wavelet Packet Modulation for Wireless Communication", the Third Advanced International Conference on Telecommunications 2007, AICT, Doi:/10.1109/AICT.2007, (2007), pp. 19-21.

[12] J. M. Lina, "Complex Daubechies Wavelets: Filter Design and Applications", Springer US, DOI: 10.1007/978-1-4020-7975-7_7 pp. 95-112.

[13] L. Gagnon, J. M Lina and B. Goulard, "Application of Complex Daubechies", Wavelets to Numerical Simulation of a Nonlinear Signal Propagation Model IEEE Seventh SP Workshop on Statistical Signal and Array Processing, 1994, DOI: 10.1109/SSAP.1994.572491, (1994).

[14] Y. Ben-Ezra and B.I. Lembrikov, Application of Complex Wavelet Packet Transform (CWPT) in Coherent Optical OFDM (CO-OFDM) Communication Systems, Chapter 6, http://dx.doi.org/10.5772/59054

[15] C. V. Bouwel, J. Potemans, S. Schepers, B. Nauwelaers and A.V. de Capelle, "Wavelet Packet Based Multicarrier Modulation", Proc. IEEE Benelux Symposium on Communications and Vehicular Technology, Leuven, Belgium, (2000). 
[16] H. M. N. Mohamed, N. S. Kamel and V. J. Jagadish, Investigation of Using Dual Tree Complex Wavelet Transform (DT-CWT) to Improve the Performance of OFDM System, Engineering Letters, 20:2, Advance online publication, (2012).

[17] M.U. Rehman, S. Wang, Y. Liu, S. Chen, X. Chen and C. G. Parini, "Achieving High Data Rate in Multiband-OFDM UWB Over Power-Line Communication System", IEEE Transactions on Power Delivery, DOI: 10.1109/TPWRD.2012.2193902, vol. 27, Issue 3, (2012), pp. 1172-1177.

[18] B. A. Balakrishnan, J. R. Foerster and A. Dabak, "Design of a multiband OFDM system for realistic UWB channel environments", IEEE Trans. Microwave Theory \& Techniques, DOI: 10.1109/TMTT.2004.834184, vol. 52, Issue 9, (2004), pp. 2123-2138.

[19] N.G. Kingsbury, "The dual-tree complex wavelet transform: A new technique for shift Invariance and directional filters", Proc. 8th IEEE DSP Workshop Utah, Doi:10.1.1.51.1213, (1998).

[20] N. G. Kingsbury, "The dual-tree complex wavelet transform: a new efficient tool for OFDM restoration and enhancement", Proc. EUSIPCO 98, Rhodes, (1998), pp. 319-322.

[21] T. Kaiser, A. WilzeckBerentsen, M. and Rupp, M. Prototyping for MIMO systems- an overview, Proceedings of 12th European Signal Processing Conference (EUSIPCO '04), Vienna, Austria, (2004), pp. 681-688. 
International Journal of Grid and Distributed Computing

Vol. 10, No. 10 (2017) 